\title{
ESTRATÉGIAS DE CUIDADO À SAÚDE MENTAL DO TRABALHADOR DURANTE A PANDEMIA DA COVID-19 uma experiência na atenção primária à saúde
}

\author{
MENTAL HEALTH CARE STRATEGIES FOR WORKERS \\ DURING THE COVID-19 PANDEMIC \\ an experience in Primary Health Care
}

Túlio Romério Lopes Quirino

(iD) http://orcid.org/oooo-0oo2-3136-4777 Prefeitura do Recife, Doutor em Psicologia

Luana Padilha da Rocha

(iD) http://orcid.org/oooo-0002-7879-8708 Prefeitura do Recife, Mestra em

Enfermagem e Educação

Maria Soraida Silva Cruz

(D) http://orcid.org/oooo-ooo1-6894-9536 Prefeitura do Recife, Mestra em Saúde da

Criança e do Adolescente

Bruna Leitão Miranda

(D) http://orcid.org/oooo-0002-5588-0362

Prefeitura do Recife, Especialista em

Psicologia no Âmbito da Saúde Mental

\author{
Janaína Gabriela Coêlho de \\ Araújo \\ D http://orcid.org/oooo-0003-3505-3797 \\ Prefeitura do Recife, Mestra em \\ Gerontologia \\ Robélia do Nascimento Lopes \\ http://orcid.org/oooo-0002-0289-9713 \\ Prefeitura do Recife, Especialista em \\ Serviço Social e Políticas de Proteção Social \\ Suênia Xavier Gonçalves \\ http://orcid.org/oooo-ooo1-5846-6338 \\ Prefeitura do Recife, Especialista em \\ Residência Multiprofissional em Saúde \\ da Família
}

\section{RESUMO}

A pandemia vem provocando sintomas de depressão, ansiedade e estresse na população em geral e também nos profissionais de saúde. A Atenção Primária à Saúde (APS) tem papel central no enfrentamento da Covid-19 e os seus trabalhadores estão sujeitos ao sofrimento mental, o que enfatiza a importância de ações voltadas ao seu bem-estar. Este estudo consiste numa sistematização de experiência, cujo objetivo é descrever estratégias de cuidado à saúde mental do trabalhador da APs durante a pandemia da Covid-19. Estas foram desenvolvidas por uma equipe do Núcleo Ampliado de Saúde da Família e Atenção Básica (Nasf-AB), na cidade do Recife. Apesar da demanda de cuidado à saúde do trabalhador (ST) da linha de frente contra a pandemia, $O$ cuidado com sua saúde mental representa uma lacuna nos protocolos de manejo da Covid-19, no Recife. Assim, o Nasf-AB viu a necessidade de resgatar 
o cuidado dos profissionais das equipes apoiadas, cuja retomada iniciou em abril de 2020. As estratégias de cuidado ofertadas são: escuta qualificada, auriculoterapia e massoterapia. A escolha da prática é livre e os encontros acontecem semanalmente. Outra estratégia foi a criação do Jornal das Flores. Essas estratégias obtiveram boa aceitação dos profissionais e o cuidado continuado vem favorecendo o melhor desempenho nas atividades cotidianas pelo alívio da dor, ansiedade e estresse. A experiência narrada sinaliza a importância de novas formas de abordar a sT e a necessidade de que tais espaços de cuidado sejam reconhecidos como dispositivos essenciais aos movimentos de retomada da rotina e superação das marcas deixadas pela Covid-19.

Palavras-chave: Pandemia; Atenção Primária à Saúde; Saúde do Trabalhador; Saúde Mental; Empatia.

\section{ABSTRACT}

The pandemic has been causing symptoms of depression, anxiety, and stress in the general population and also withinin the health professionals. Primary Health Care (PHC) plays a central role in coping with Covid-19 and its workers are subject to mental suffering, which emphasizes the importance of actions aimed at their well-being. This study aims to describe strategies for mental health care of PHC workers during the Covid-19 pandemic. Said strategies were developed by a team of the Family Health and Primary Care Extended Centers (Nasf-AB) in the city of Recife. Despite the demand for frontline worker health care (WHC) against the pandemic, mental health care represents a gap in Covid-19 management protocols in Recife. Thus, the Nasf-AB saw the need to restore health care for the professionals of the supported teams, which resumed in April 2020. The care strategies offered are qualified listening, auriculotherapy and massage therapy. Practice choice is free and meetings take place weekly. Another strategy was the creation of the Jornal das Flores newspaper. These strategies have good acceptance and that continuing healthcare has been resulting in better performance in daily activities by relieving pain, anxiety, and stress. The narrated experience indicates the importance of new ways of addressing WHC and the need for care spaces to be recognized as essential for the routine resumption and overcoming the marks left by Covid-19.

Keywords: Pandemic; Primary Health Care; Occupational Health; Mental Health; Empathy.

\section{INTRODUÇÃO}

A pandemia da Covid-19 gerou a necessidade da implementação de medidas não farmacológicas de intervenção, entre elas o distanciamento social, para conter o crescimento exponencial do número de pessoas infectadas, haja vista a alta transmissibilidade do vírus, o grande número de casos, a inexistência de vacina ou 
tratamento medicamentoso comprovado, a testagem insuficiente da população e a duração prolongada dos quadros clínicos, com grande potencial de agravamento (CRUZ et al., 2020; JACKSON FILHO et al., 2020).

Diante da situação de confinamento e isolamento, traduzida numa percepção de quarentena generalizada, com a adoção de medidas voltadas ao comportamento seguro e ao cumprimento de regras para o autocuidado, acentuou-se outra preocupação: os problemas relacionados à saúde mental. Além dos impactos psicológicos diretamente relacionados à Covid-19, os efeitos negativos das medidas de distanciamento social incluem sintomas de estresse pós-traumático, confusão e raiva. Associam-se a isso preocupações com a escassez de suprimentos, perdas financeiras e a exposição constante a notícias sobre a doença em mídias sociais (CRUZ et al., 202O; SCHIMIDT et al., 2020).

Pode-se mencionar, ainda, a proliferação de informações imprecisas e sem comprovação científica (fake news), que provocam desconfianças, incertezas e manipulação, podendo gerar respostas ansiogênicas e depressivas, o que levou a Organização Pan-Americana de Saúde a identificar, também, uma “infodemia” (OPAS, 2020).

Ante o cenário de pandemia, o medo de ser infectado por um vírus de alta disseminação e significativo potencial de mortalidade, com origem, natureza e curso pouco conhecidos, afeta o bem-estar psicológico das pessoas. Sintomas de depressão, ansiedade e estresse, além de acometerem a população em geral, afetam, também, os profissionais de saúde. Durante surtos epidêmicos e pandemias, esses profissionais trabalham por muitas horas, sem pausas e sob grande pressão, o que pode levá-los à fadiga e exaustão (SCHIMIDT et al., 202O; PRADO et al., 202O; HELIOTERIO et al., 2020).

No contexto da Covid-19, os profissionais de saúde envolvidos no diagnóstico, tratamento e atendimento de pacientes infectados podem apresentar altos índices de sofrimento psíquico, como medo, ansiedade, depressão, angústia e sono prejudicado. Além do receio do próprio contágio, fatores como a preocupação com o risco de infectar outras pessoas, inclusive a própria família, o que leva ao afastamento desses profissionais dos seus parentes e amigos; a sobrecarga de trabalho; as ameaças e agressões, realizadas por pessoas que buscam atendimento e não conseguem, devido à limitação de recursos; as limitações no acesso a equipamentos de proteção individual (EPI); as mudanças frequentes nos protocolos de atendimento, devido às novas descobertas sobre a 
doença; entre outros, podem ser gatilhos para o desencadeamento e/ou intensificação de problemas mentais (PRADO et al., 2020; sCHIMIDT et al., 2020).

Uma vez que a preocupação atual das iniciativas em políticas públicas de saúde, como não poderia deixar de ser, encontra-se centrada nos cuidados à população, na regulamentação de leitos e no monitoramento de casos suspeitos, a situação de esgotamento dos profissionais de saúde tem recebido pouca atenção. No entanto, intervenções que busquem atenuar o sofrimento psíquico desses trabalhadores devem ser empregadas precocemente, de acordo com o tipo e a dinâmica de cada serviço, incluindo as Unidades Básicas de Saúde (SBMFC, 2020).

A despeito dos investimentos na atenção hospitalar, com vistas à ampliação de leitos, principalmente em Unidades de Terapia Intensiva, a Atenção Primária à Saúde (APS) também tem papel central no enfrentamento da Covid-19. Ela está ancorada nos princípios de proteção da saúde, prevenção e controle de doenças, sendo capaz de colaborar estrategicamente tanto na redução dos riscos de transmissão da doença, a partir do diagnóstico precoce, e no acompanhamento e monitoramento individual e familiar, quanto nas ações de educação em saúde e na colaboração junto aos demais níveis de atenção. A APs constitui, assim, uma rede protetora para a segurança e a qualidade do cuidado, especialmente, no caso de uma demanda comunitária de epidemia viral. Desse modo, inseridos neste contexto pandêmico, os trabalhadores da APs também estão sujeitos a situações de pressão psicológica e sofrimento mental (RIBEIRO et al., 2020; sOEIRO et al., 2020).

Apesar de grande parte dos problemas psicossociais ser, até mesmo, esperada diante de situações adversas, é essencial a realização de ações voltadas à saúde mental, ao apoio psicossocial e ao bem-estar dos trabalhadores durante e após uma epidemia. Esses problemas precisam ser trabalhados em conjunto com as próprias redes tradicionais de solidariedade, como família, amigos, comunidade, e entre os próprios colegas de trabalho (BRASIL, 2020a). Diante dessas questões, este artigo tem como objetivo debater estratégias de cuidado à saúde mental do trabalhador de saúde da APS, no cenário da pandemia da Covid-19. Essas estratégias foram desenvolvidas por uma equipe do Núcleo Ampliado de Saúde da Família e Atenção Básica (Nasf-AB) na cidade do Recife, Pernambuco, com objetivo de ofertar suporte psicossocial aos profissionais que têm atuado na linha de frente desses serviços territoriais. 


\title{
CAMINHO METODOLÓGICO
}

A escrita deste texto brota da integralização de vivências interprofissionais desenvolvidas por uma equipe do Nasf- $\mathrm{AB}$ atuante no cenário da APS do município do Recife. Resulta, portanto, do resgate de situações e circunstâncias laborais, desenvolvidas no cotidiano da atenção à saúde, postas à reflexão coletiva em um exercício ordenado de problematização do vivido. Nesses termos, o presente estudo consiste em uma sistematização da experiência, cuja inspiração metodológica baseia-se na proposta de Holliday (2006), definida como método para organizar, analisar e comunicar vivências, possibilitando a sua interpretação crítica.

À diferença de outras abordagens, que se limitam à descrição de acontecimentos de maneira ordenada, $\mathrm{o}$ ato da sistematização não se restringe a "narrar experiências". Mais do que isso, ele busca proporcionar uma compreensão aprofundada das experiências desenvolvidas, situando-se em um caminho intermediário entre a descrição e a teoria. Segundo Holliday (2006), o ordenamento e a reconstrução de uma experiência possibilitam a sua interpretação crítica, permitindo descobrir e/ou explicitar a lógica do processo vivenciado, os fatores que o influenciam, como eles se relacionam entre si e por que foram feitos desse modo.

Para tanto, o relato deve ser sistematizado em cinco tempos: ponto de partida; perguntas iniciais; recuperação do processo vivido; reflexão de fundo e pontos de chegada. Apesar dessa linha orientadora, a sistematização não precisa necessariamente seguir essa ordem ou abordar todos esses aspectos, pois o seu desenvolvimento depende de diversos fatores que incidem na multiplicidade

\author{
ce \\ a organização de cuidados \\ primários em saúde revela-se \\ como potencial atributo na \\ produção de respostas exitosas \\ diante de crises sanitárias
}


de experiências existentes. A experiência aqui sistematizada observa a proposta de Holliday (2006) e se desenvolve em três momentos: "contextualização espaço-temporal" (ponto de partida); "comunicação do vivido" (reflexão de fundo); e "aprendizados e ressonâncias" (ponto de chegada).

\section{CONTEXTUALIZANDO O CENÁRIO: A REORGANIZAÇÃO DA APS COMO PONTO DE PARTIDA}

Constituindo uma das principais características dos sistemas de saúde universais, como o brasileiro, a organização de cuidados primários em saúde revela-se como potencial atributo na produção de respostas exitosas diante de crises sanitárias. Isso porque tal forma de organização garante a oferta de serviços de saúde mais próximos e adequados às necessidades dos grupos populacionais. Essa proximidade facilita o acompanhamento e o monitoramento da situação de saúde dessas populações e, em consequência, a intervenção precoce diante de adoecimentos, minorando as possibilidades de agravamento.

No contexto do Sistema Único de Saúde, a APs está estruturada como porta de entrada preferencial dos usuários. Ela tem a Estratégia Saúde da Família (ESF) como dispositivo prioritário para coordenar o cuidado e ordenar os fluxos assistenciais que delineiam os deslocamentos na rede de saúde, com vistas a garantir a integralidade da atenção e a universalização do acesso aos serviços de saúde. Essa lógica de funcionamento acompanha a dimensão territorial das intervenções em saúde e a abordagem comunitária, como aspectos que aproximam a produção de respostas adequadas às demandas locais, revelando-se, desde aí, a efetividade do trabalho desempenhado pelas equipes da APs.

Com a emergência da Covid-19, haja vista a magnitude da doença e os poucos conhecimentos sobre recursos efetivos ao seu enfrentamento, bem como a importância do trabalho desenvolvido pela APs, foi imperativa a reorganização dos processos de trabalho e a adoção de medidas mais rígidas de biossegurança, dado o reconhecimento de que o exercício das atividades laborais aderido às condições de trabalho constitui fonte potencial de exposição ao vírus (JACKSON FILHO et al., 2020).

Diante da pandemia, observou-se, em todo o país, uma dupla tendência na organização da APS: (1) a priorização da oferta clínico-assistencial, com consequente diminuição da abordagem preventiva e de promoção da saúde; e (2) a criação temporária de equipes, fluxos e/ou rotinas específicas para o atendimento de 
sintomáticos respiratórios. Essa organização foi amparada nas recomendações do Ministério da Saúde, estabelecidas desde as primeiras versões do protocolo de manejo clínico do coronavírus na APS (BRASIL, 2020b).

A primeira tendência de organização envolveu a ampliação dos atendimentos clínicos no âmbito dos serviços, os quais se voltaram especificamente à manutenção de rotinas assistenciais para a continuidade no acompanhamento dos chamados "grupos prioritários". Desses grupos participam as pessoas com doenças crônicas, os idosos, as gestantes, as puérperas e as crianças com idade de até os cinco anos. Logo, a atenção a estes grupos passou a se restringir às consultas de rotina, individuais, e com marcação prévia.

No caso dos atendimentos domiciliares, as medidas de distanciamento social adotadas pelos municípios colocaram restrições à sua realização. Foram mantidas as visitas apenas para os casos considerados de estrita necessidade e desde que fossem respeitadas as medidas de proteção à saúde de profissionais e usuários. Também em razão do distanciamento social, as atividades de cunho educativo, como grupos e salas de espera, foram suspensas, assim como a maior parte das atividades no território. Nesse contexto, os profissionais ficaram praticamente restritos às dependências físicas das unidades de saúde, numa lógica que pareceu subverter a atuação comunitária e territorial das equipes da APS.

Por outro lado, iniciativas de acompanhamento remoto foram colocadas como opções que podiam ser observadas pelas equipes, por meio de contatos via telefone ou aplicativos de mensagem. No entanto, tais iniciativas eram também dedicadas ao monitoramento de casos suspeitos e/ou ao acompanhamento dos já referidos grupos prioritários, principalmente aqueles considerados como população de risco de adoecimento pela Covid-19.

A segunda tendência de reorganização da APs está relacionada à restrição das atividades das equipes ao espaço físico das unidades de saúde, onde os profissionais passaram a desenvolver uma atuação específica para o atendimento de sintomáticos respiratórios. Nesse caso, caracteriza-se a criação de equipes temporárias, com processo de trabalho voltado estritamente ao diagnóstico, manejo clínico e monitoramento dos casos. Essa organização está amparada nas orientações do Ministério da Saúde para realização do Fast-Track, metodologia para triagem e abordagem clínica utilizada em emergências, com vistas ao atendimento rápido de pessoas sintomáticas, de modo a evitar sua circulação nos serviços e a exposição de outras pessoas (BRASIL, 2020b). 


\section{os profissionais, em muitos casos, passaram a se afastar de seu convívio familiar no intuito de proteger seus parentes dos riscos de contaminação}

Como exemplo dessa ação, pode-se citar a definição de equipes para as Unidades Provisórias Centralizadas de APs, em Recife, Pernambuco. Em algumas unidades, essas equipes têm o apoio dos serviços de teleatendimento e/ou teleorientação, metodologias adotadas para evitar que pessoas com sintomas respiratórios leves percorram os territórios e se desloquem para os serviços de saúde, prevenindo a infecção de outras pessoas. Assim, são acompanhadas em domicílio, durante um período de 7 a 14 dias, sendo orientadas a buscarem os serviços de saúde se os sintomas se agravarem.

Esse novo cenário posto à APs, como não poderia deixar de ser, apresentou impactos importantes nos processos de trabalho desenvolvidos pelas equipes. Logo, em resposta à necessidade de reorganização dos processos de trabalho, foram emitidas recomendações de novos arranjos e rotinas institucionais e tomadas medidas de biossegurança e de manejo clínico da Covid-19.

A terceira reorganização ocorreu quando o município do Recife, seguindo a tendência nacional e observando as orientações normativas disponibilizadas pelo Ministério da Saúde, lançou, no mês de março de 2020, a primeira versão do seu Protocolo de Assistência e Manejo Clínico na Atenção Primária à Saúde (RECIFE, 2020a). Esse documento tinha por objetivo nortear as atividades a serem desenvolvidas nas Unidades de Saúde da Família (USFs), visando à redução do fluxo de usuários e do tempo de permanência dos mesmos nas unidades de saúde.

O protocolo trouxe orientações quanto ao atendimento dos pacientes sintomáticos respiratórios, à suspensão dos atendimentos de rotina, com exceção do acompanhamento de alguns casos 
elencados como prioritários, e à suspensão das atividades coletivas. O documento versava ainda sobre uma nova forma de organização do processo de trabalho nas USFs.

$\mathrm{Na}$ quarta atualização do protocolo, com o intuito de diminuir os riscos de disseminação do vírus nos postos de trabalho, o município realizou novo ordenamento na APs, destacando algumas unidades de saúde como referência para atendimento exclusivo aos usuários que manifestassem sintomas respiratórios, as denominadas "Unidades de Referência para Atendimento aos Sintomáticos Respiratórios". Nessas unidades, atuavam profissionais da ESF e das Unidades Básicas Tradicionais (UBT), em esquema de rodízio, sob designação dos Distritos Sanitários aos quais estavam vinculados (RECIFE, 2020b). Apesar da publicação do protocolo e de suas posteriores atualizações, a reorganização do trabalho para o acolhimento das demandas relacionadas à pandemia e a necessidade de reestruturação das atividades cotidianas pouco foram discutidas junto às equipes de Saúde da Família (SF).

Jackson Filho e colaboradores (2020) afirmam que, mesmo entre profissionais de saúde diretamente ligados aos cuidados a pacientes, pouco se discute sobre condições e organização do trabalho. Prevalecem os protocolos com recomendações individuais, como as medidas de higiene e o uso de EPIs: todas elas fundamentais, mas insuficientes para o controle geral da disseminação e da exposição ao vírus. Ademais, pouco se aborda, por exemplo, as condições de trabalho precarizadas, a higiene inadequada dos postos de trabalho, as jornadas de trabalho exaustivas, a falta de treinamento e, inclusive, a insuficiência ou indisponibilidade de EPIs.

Diante do cenário desenhado pela pandemia, observamos que o deslocamento dos profissionais de saúde de suas unidade de atuação trouxe como consequência o aumento de demanda reprimida e a sobrecarga de trabalho para algumas equipes. Somando-se a isso, os espaços de reunião de equipe, que antes se encontravam organizados e em funcionamento sistemático, foram suspensos. Tais espaços serviam de ponto de encontro entre os profissionais para discussões e planejamento das ações no território, além de serem importantes para o fortalecimento de vínculos.

Essas considerações podem ser aplicadas aos diversos cenários vividos pelas equipes de $\mathrm{SF}$, e não se afastam do contexto vivenciado pelos profissionais das ESF apoiadas pela equipe do Núcleo Ampliado de Saúde da Família e Atenção Básica (Nasf-AB), cuja experiência está aqui sendo posta em análise. Observa-se, ainda, que os profissionais, em muitos casos, passaram a se afastar de seu 
convívio familiar no intuito de proteger seus parentes dos riscos de contaminação; sem contar a transmutação e intensificação dos processos de trabalho, que, por si só, já seriam capazes de gerar um potencial fator de adoecimento. Em razão desse distanciamento de parentes e amigos, como já apontado, sentimentos de fragilidade e situações de sofrimento mental eram potencializados e repercutiam no seu estado de saúde. É a partir desse contexto que passaremos a narrar, a seguir, propostas e experiências de cuidado desenvolvidas para enfrentar as situações de adoecimento de trabalhadores de saúde no cotidiano da APs.

\section{COMUNICAÇÃO DO VIVIDO: SISTEMATIZANDO PRÁTICAS DE CUIDADO AO PROFISSIONAL DE SAÚDE}

O cotidiano de trabalho do Nasf-AB é permeado pelo dinâmico conjunto de práticas de saúde desenvolvidas junto às equipes da ESF, ensejando a ampliação da clínica na APS e da capacidade de resolutividade de suas demandas. Tendo o apoio matricial como referencial, posto à sua organização, esse fazer multiprofissional mobiliza diferentes ferramentas tecnológicas de cuidado que ora se voltam para a garantia da assistência clínica e o desenvolvimento do cuidado em saúde para usuários e coletividades, ora para o suporte técnico e pedagógico aos profissionais da ESF, auxiliando-os na condução de casos e construindo responsabilidade compartilhada no acompanhamento do território de sua referência.

O desenvolvimento dessa forma de trabalho demanda constantes discussões e reflexões, além de novas pactuações e reorientações. Os contextos comunitários possuem características singulares em suas formas de organização da vida, as quais não são, de modo algum, estáticas, gerando cotidianamente novas necessidades de saúde que devem ser acolhidas e ressignificadas pelas equipes da APs, dentre elas o Nasf-AB. Logo, em nossa compreensão, como equipe Nasf- $\mathrm{AB}$, que toma aqui a própria experiência como objeto de reflexão, a inventividade e a proposição de iniciativas, considerando as especificidades de cada território, constituem posturas inerentes à efetivação do apoio matricial.

Ao longo de mais de seis anos de atuação em um território sanitário situado em uma região periférica do município, cuja localização geográfica remete ao sul, temos construído e reconstruído rotinas laborais junto a, pelo menos, nove equipes da ESF. Nesse período, foram acumuladas diversas ações de cuidado aos usuários e seus familiares, mas também aos profissionais que atuam nos 
serviços da APs, que nos reconhecem como referências de apoio. Esse conjunto de atividades, de caráter individual e/ou coletivo, pontual ou sistemático, reflete a bricolagem tecida no trabalho interprofissional realizado nesse contexto de atenção, em que se combinam atendimentos clínicos, suporte ao cuidado territorial, grupos educativos e terapêuticos, processos de educação permanente em saúde e de articulação intersetorial, dentre outras ações.

Fazendo parte deste rol de atuações, a atenção à saúde do trabalhador de saúde foi alçada à condição de uma das rotinas a serem garantidas. Desse modo, desde meados de 2016, temos desenvolvido, de modo itinerante, o espaço Cuidando do Cuidador, cujo objetivo é cuidar da saúde mental dos profissionais das equipes de ESF, uma vez que, desde a nossa inserção nesse território, observamos que alguns profissionais apresentavam certa desmotivação, queixas de adoecimento e afastamentos do trabalho. Diante desses fatos, mobilizamo-nos para pensar estratégias de cuidado para esses trabalhadores, sendo elaborada e apresentada a proposta nos espaços de reunião entre equipes Nasf-AB e da SF, com plena aceitação.

O Cuidando do Cuidador passou a ser desenvolvido por meio de encontros sistemáticos com pactuação prévia entre as equipes envolvidas. Esses encontros ocorriam em espaços públicos, de preferência, abertos à livre circulação, como praças, parques e museus. Para cada encontro, os profissionais da EsF escolhiam o local de sua realização e a equipe Nasf-AB organizava as atividades e mediava os encontros. Como estratégias de intermediação das atividades, eram mobilizadas técnicas grupais, além de jogos e atividades físicas, de preferência, apostando na ludicidade como ferramenta para favorecer o fortalecimento de vínculos e a descontração entre os membros das equipes. A periodicidade desses encontros variava de acordo com a necessidade de cada equipe apoiada.

A proposta teve boa adesão inicial, porém, com o passar do tempo, a participação e o envolvimento dos profissionais da ESF foram diminuindo, levando os encontros a serem cada vez mais espaçados, até terem sua oferta finalizada pela equipe Nasf-AB, em dezembro de 2019.

Diante da pandemia da Covid-19, fatores como a sensação de vulnerabilidade, o temor de que algo de ruim aconteça a si e aos outros, o aumento das demandas de trabalho nos serviços e a perda de controle sobre os acontecimentos são aspectos que trazem ao trabalhador de saúde repercussões importantes, levando a uma importante sobrecarga emocional no trabalho, com impactos no desgaste físico e mental (HELIOTERIO et al., 2020). 
Questões como essas nos demonstraram a necessidade de retomar as ações de cuidado aos trabalhadores de saúde, ainda que houvesse o impeditivo de resgatar as propostas já desenvolvidas anteriormente, em observância tanto às disposições normativas quanto à não manutenção de atividades coletivas no âmbito da APs. $O$ cenário vivido nesse cotidiano assistencial revelava o permanente estado de tensão entre as equipes de saúde, seja pelos temores do adoecimento, seja pelas dores físicas oriundas do exercício do trabalho, ou mesmo pelas inseguranças e incertezas do momento vivido. Diante disso, não vimos outra alternativa a não ser resgatar o Cuidando do Cuidador, mas garantindo uma nova formatação, para que pudéssemos desenvolver a ação com a segurança necessária.

A retomada da atividade foi iniciada em meados de abril de 2020, com a oferta de práticas de caráter individual, dentre elas a escuta qualificada, a auriculoterapia e a massoterapia. A definição dessas práticas levou em consideração os núcleos e saberes específicos de profissionais integrantes da equipe Nasf-AB, buscando-se aquelas que melhor pudessem se adequar às necessidades relatadas pelos profissionais das ESFS apoiadas.

A ocorrência dos encontros de cuidado foi definida com periodicidade semanal, e a participação é de livre demanda para os profissionais interessados, que precisam informar previamente seu interesse ao terapeuta responsável, para os devidos ajustes de agenda; haja vista a quantidade de atendimentos desenvolvidos em cada período. Vale salientar que não apenas os profissionais de saúde, mas também trabalhadores de serviços gerais, recepção e vigilância, podem ter acesso às práticas. Os locais definidos para a realização dos atendimentos foram as dependências das próprias USFs, com a utilização dos consultórios disponíveis em cada momento programado. Observar essa estrutura foi importante para garantir o sigilo e a privacidade dos participantes, condição essencial para favorecer os relatos e estabelecer a confiança e o vínculo entre o profissional-terapeuta e a pessoa atendida.

A auriculoterapia (AT) faz parte do rol de práticas integrativas e complementares (PICs) inseridas no cotidiano do sUs (BRASIL, 2015). Tendo como base a Medicina Tradicional Chinesa (MTC), a auriculoterapia se desenvolve através da estimulação de pontos específicos do pavilhão auditivo externo, visando ao tratamento de diversas condições de saúde, dentre elas a dor crônica (ARTIOLI, TAVARES, BERTOLINI, 2019), ansiedade e estresse (JALES et al., 2019). 


\section{A intervenção na crise deve alicerçar-se no acolhimento do sujeito e de suas emoções}

Sendo essas as queixas mais comuns observadas entre os profissionais das ESFs apoiadas, foi desenvolvido um protocolo de orientação prática, com pontos de referência, a fim de melhor acompanhar a evolução dos sintomas. Na equipe Nasf-AB, dispomos de três profissionais que possuem formação e habilidades no uso da AT (duas fisioterapeutas e uma terapeuta ocupacional), que organizaram suas rotinas para ofertar essa modalidade de cuidado.

A dor é uma condição prevalente entre os trabalhadores da saúde, estando relacionada a fatores como sobrecarga emocional e física, além de precárias condições no ambiente de trabalho. Estudos têm apontado que categorias profissionais, no contexto da APS, têm sido afetadas com distúrbios musculoesqueléticos, principalmente dentistas e técnicos de saúde bucal, enfermeiros e técnicos de enfermagem, e agentes comunitários de saúde (BARBOSA, ASSUNÇão, ARAÚjO, 2012). Em confirmação a esses estudos, durante nossa vivência prática, observamos que as maiores queixas relatadas estão relacionadas às dores tensionais, principalmente nas regiões cervical e lombar. Em vista disso, a massoterapia foi outra prática ofertada aos trabalhadores das USFs, sendo executada pelas profissionais do Núcleo da Fisioterapia, que já tinham experiência com técnicas de massagem, vivenciadas desde a graduação e aprimoradas em cursos de aperfeiçoamento.

A escuta qualificada tem seu pilar na Política Nacional de Humanização (PNH) (BRASIL, 2013). Constitui um dispositivo de cuidado por meio do qual é possível uma compreensão abrangente e empática das necessidades apresentadas pelo sujeito. A partir disso, tem como propósito ajudar a pessoa na identificação de estratégias para enfrentamento da condição relatada e, até mesmo, para estruturar um plano terapêutico de cuidado (santos, 2019). Dessa forma, com a sua oferta no âmbito do Cuidando do 
Cuidador, os trabalhadores encontram um espaço protegido para relatar e dialogar sobre seus problemas, além de receber suporte emocional, com a psicóloga da equipe, e orientações quanto à reorganização das ocupações frente à nova rotina, com a terapeuta ocupacional.

Entendemos que todas as pessoas possuem forças e habilidades para lidar com os desafios da vida. No entanto, em contextos de crise, alguns indivíduos podem precisar de maior apoio psicossocial (BRASIL, 2020a). Nesse contexto, as práticas de cuidado junto aos profissionais tornam-se fundamentais. A intervenção na crise deve alicerçar-se no acolhimento do sujeito e de suas emoções, de forma sensível, com foco no enfrentamento eficaz, na resolução de problemas, na esperança e nos pensamentos positivos, buscando respostas psicoemocionais adaptativas e saudáveis (PRADO et al., 2020).

Os cuidados psicológicos junto aos trabalhadores podem ser realizados presencialmente, virtualmente, através de plataformas on-line e ligações telefônicas, ou a partir de cartilhas e de outros materiais informativos (sCHIMIDT et al., 2020). Desse modo, outra estratégia utilizada na organização de práticas de cuidado voltadas aos profissionais de saúde durante a pandemia foi a criação do Jornal das Flores, cujo foco está no trabalhador e em sua valorização (Fig. 1).

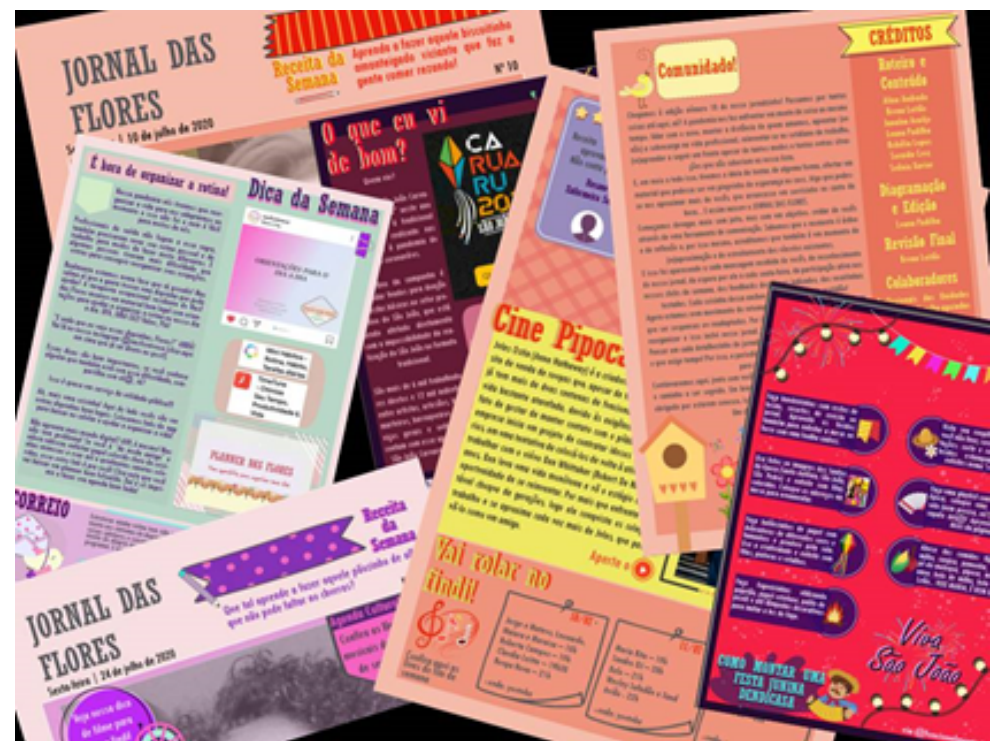

Fonte: Elaborado pelos autores, 2020. 
Essa publicação é composta por sessões temáticas, atualizadas semanalmente, em conexão com os acontecimentos cotidianos no cenário de atuação das equipes. Constituem a estrutura do jornal: (1) Click da semana (registro fotográfico de algum trabalhador das USFs, acompanhado por uma entrevista com o mesmo); (2) Receitas; (3) Sugestões de filmes; (4) Agenda cultural com as lives da semana; (5) Correios (espaço para troca de recados entre os profissionais); (6) Dicas (relacionadas ao autocuidado e/ou a ideias para executá-lo durante o período de quarentena, com foco na saúde e bem-estar); e (7) O que eu vi de bom? (seção que traz acontecimentos positivos daquela semana). O jornal tem periodicidade semanal e sua divulgação ocorre por meio dos grupos de WhatsApp usados entre a equipe Nasf-AB e as Esfs.

O Jornal das Flores obteve ótima aceitação entre as equipes e atingiu o objetivo de levar um pouco de descontração e leveza aos protagonistas do cuidado em saúde durante esse período de pandemia. Essa atividade proporciona certo alívio cômico e faz circular mensagens positivas que possibilitam suporte motivacional, além de contribuir para a integração das equipes e para o fortalecimento de vínculos interprofissionais.

\section{APRENDIZADOS E RESSONÂNCIAS: RESGATANDO O CUIDANDO DO CUIDADOR EM TEMPOS DE PANDEMIA}

As mudanças geradas pela pandemia no cotidiano dos trabalhadores das equipes de SF, tanto na vida pessoal quanto na vida profissional, levaram muitos profissionais ao desgaste físico e emocional. O medo e as incertezas passaram a fazer parte das narrativas cotidianas dos trabalhadores dos serviços, e muitos foram os afastamentos por motivos de saúde durante esse período. Apesar da existência de importante demanda para a realização de abordagens à saúde do trabalhador que está na linha de frente contra a pandemia, o cuidado com a sua saúde mental teve pouca atenção, com lacunas quanto à implementação de propostas de ação para fornecer esse tipo de atenção nos protocolos de manejo da Covid-19 no município.

De modo geral, na vigência de pandemias, a saúde física da população e o combate ao agente patogênico são o objeto de trabalho prioritário de gestores e profissionais da saúde, deixando as implicações sobre a saúde mental negligenciadas ou subestimadas. Todavia, as ações de manejo dos impactos psicológicos da pandemia e suas repercussões futuras não podem ser menosprezadas, principalmente em se tratando de profissionais que atuam 
em contato direto com pessoas infectadas pelo vírus. Isso posto, é essencial o desenvolvimento de estratégias de cuidado que possam envolver a orientação sobre os sintomas psicológicos que os profissionais de saúde podem apresentar nesse contexto, como estresse, depressão, ansiedade e insônia, além de promover medidas de enfrentamento e autocuidado (sCHIMIDT et al., 2020).

Em nosso território de atuação, nos deparamos com equipes de sF fragilizadas e desgastadas diante desta nova realidade assistencial, revelando a necessidade de espaços voltados à escuta e à fala, além de serem observadas queixas de dores corporais, dificuldades para conciliar o sono e para a reorganização de rotinas relacionadas ao trabalho e à vida pessoal. Foi esse panorama que nos demonstrou a necessidade de resgatar o trabalho de cuidado voltado aos profissionais das equipes apoiadas, com a finalidade de fortalecê-las no enfrentamento da conjuntura criada pela pandemia.

Nesses termos, o resgate do Cuidando do Cuidador, ante à pandemia da Covid-19, trouxe a necessidade de readequação de sua formatação, transmutando as práticas ofertadas e a lógica de trabalho estabelecida até então. $\mathrm{O}$ que antes se desenvolvia como espaço coletivo e externo ao serviço de saúde precisou ser individualizado e restrito ao ambiente físico das USFs, obedecendo às medidas sanitárias preconizadas. Com isso, voltamo-nos para a identificação, mesmo entre núcleos específicos, das potencialidades existentes em nosso próprio campo de atuação, desvelando em equipe habilidades e competências práticas e interprofissionais que pudessem contribuir com o fortalecimento dos trabalhadores de saúde neste período de crise.

Como saldo desse processo, a escuta qualificada, a auriculoterapia, a massoterapia e o Jornal das Flores emergiram no cotidiano das nossas práticas e obtiveram boa aceitação entre os trabalhadores. A oferta de cuidado continuado vem revelando resultados positivos na promoção do bem-estar individual e coletivo, favorecendo o melhor desempenho nas atividades cotidianas pelo alívio da dor, da ansiedade e do estresse. Enfatizamos, dessa forma, a importância da atenção à saúde do trabalhador para a manutenção e qualificação do cuidado, tanto de quem cuida quanto daqueles que são cuidados. Tal atenção deve se manter não apenas em cenários adversos, ou no âmbito de emergências de saúde pública, mas como uma prática regular de promoção à saúde e prevenção do adoecimento, seja físico ou emocional.

Em relação à continuidade da oferta desse espaço de cuidado no pós-pandemia, em especial no que tange à saúde mental, não 
dispomos, ainda, de um formato definido, já que as propostas de ação das equipes de APs, como já mencionamos, são dinâmicas e mutantes, pois dependem dos contextos singulares e das demandas daqueles para as quais se voltam. No entanto, reconhecemos que a sua manutenção é fundamental para os profissionais de saúde, a fim de que possam desenvolver recursos subjetivos para enfrentar os desdobramentos da pandemia sobre a situação de saúde da população sob seus cuidados.

Nessa direção, as estratégias de cuidado podem continuar voltadas para os aspectos biopsicossociais, tanto por meio de atendimentos individuais quanto através de atividades coletivas, à medida que estas puderem ser retomadas, com o intuito de resgatar e fortalecer os vínculos afetivos e promover momentos de bem-estar. Desse modo, essas estratégias poderão auxiliar na redução dos impactos negativos, tais como perdas e transformações emocionais, sociais e econômicas decorrentes da pandemia.

\section{CONSIDERAÇÕES FINAIS}

A Covid-19 tem mobilizado o enfrentamento de uma grande crise sanitária, com proporções globais, revelando impactos importantes nos sistemas de saúde de países por todos os continentes. Ao longo de mais de seis meses, as marcas dessa crise são observadas pela constatação de um grande contingente de casos confirmados, quadros graves de adoecimento, colapsos assistenciais e grande quantitativo de desfechos negativos. A tomada de medidas urgentes e rigorosas pelas estruturas de governo é imperiosa, a fim de mitigar os efeitos deletérios que a pandemia provoca na população.

No âmbito do seu enfrentamento, a APs converte-se em um dos principais lócus de intervenção. Isso se dá pela sua capilaridade assistencial, uma vez que responde pela maior parte das demandas de saúde que acometem os grupos populacionais, com amplo potencial de resolutividade. Além disso, a APS é relevante pela abordagem comunitária e territorial que desenvolve, a qual tem importante papel no acompanhamento, monitoramento, possibilidade de identificação precoce e intervenção junto a casos leves identificados da doença.

Particular atenção merecem as equipes profissionais que atuam nesse cenário. Ao constituírem o primeiro contato dos usuários com o sistema de saúde, esses atores são os primeiros que "chegam", estando na linha de frente da assistência. São, também, os últimos a "saírem", já que, pela forma de organização do seu trabalho, dão continuidade e acompanham os resultados das 
intervenções desenvolvidas em outros pontos da rede de atenção, adquirindo responsabilidade pelos desdobramentos dos processos de cuidado realizados junto aos usuários. É preciso reconhecer, também, os agentes comunitários de saúde, profissionais que nunca "saem de cena".

Logo, o acúmulo de sofrimentos relacionados ao exercício cotidiano do trabalho na APs, em geral caracterizado pelo estabelecimento de rotinas assistenciais pouco sensíveis em reconhecer e dar conta das complexas demandas que povoam os territórios comunitários, acaba somando-se à extenuante vivência laboral. A vivência atual, decorrente da emergência sanitária ocasionada pela Covid-19, é marcada por processos sistemáticos de auto e heterovigilância, pela manutenção rigorosa de protocolos de atendimento e biossegurança, incluindo a permanente (e incômoda) utilização de EPIs, e pelas inseguranças das práticas e da autorresponsabilização quanto à atualidade das intervenções. Esses e outros aspectos constituem-se como potenciais geradores de preocupação, medo, insegurança, comportamentos repetitivos e quadros ansiosos.

Apesar disso, a saúde do trabalhador não tem sido tomada como uma prioridade neste contexto, quer seja do ponto de vista das equipes gestoras locais, quer seja na perspectiva dos próprios trabalhadores de saúde, que se reconhecem mais facilmente como sujeitos cuidadores, responsáveis pelos cuidados da população adstrita ao serviço, mas pouco se veem como sujeitos que necessitam de cuidados. Nesses termos, a pandemia da Covid-19 nos faz recentrar o debate sobre a ST, uma vez que impõe o reconhecimento dos riscos ao adoecimento. Esses riscos são revelados com o registro de afastamentos de profissionais situados em grupos de risco e daqueles que tiveram confirmação de contágio pelo SarsCoV-2; são também apresentados com o registro daqueles que chegaram ao agravamento e ao desfecho negativo dos seus quadros.

No caso do sofrimento psicossocial relacionado à vivência do trabalho em um contexto de adversidades, como o atual, ainda é importante considerar os efeitos do distanciamento social, das incertezas sobre a prática profissional e do enfrentamento das perdas observadas no cotidiano, como as de colegas de trabalho, de familiares e de usuários acompanhados no território. A falta de suporte para lidar com essas questões e o consequente silenciamento das dores que acompanham a vivência das crises emocionais daí resultantes podem, com o tempo, se desdobrar em quadros de sofrimento psicológico ainda mais graves, apontando para uma desafiadora vivência pós-pandemia. 
É nesse sentido que a experiência aqui narrada, ao apresentar possibilidades de intervenção interprofissional, baseadas no estabelecimento de redes de solidariedade e colaboração entre pares, por meio do fortalecimento de espaços para o autocuidado e o cuidado do outro, entre equipes de saúde que compartilham o mesmo cotidiano assistencial, sinaliza a importância das novas formas de abordar a Saúde do Trabalhador. Esta experiência, também, mostra a necessidade de que os espaços de cuidado sejam reconhecidos como dispositivos essenciais para os movimentos de retomada das atividades presenciais e para a superação das marcas deixadas pela Covid-19. Nesse contexto, os trabalhadores da saúde devem estar situados no centro das ações de cuidado, e é por meio dele que terão a motivação necessária para dar continuidade de seu trabalho.

\section{REFERÊNCIAS}

ARTIOLI, D. P.; TAVARES, A. L. de F.; BERTOLINI, G. R. F. Auriculoterapia: neurofisiologia, pontos de escolha, indicações e resultados em condições dolorosas musculoesqueléticas: revisão sistemática de revisões. $B r J P$, São Paulo, v. 2, n. 4, p. 356-361, dez. 2019. DOI: https://doi.org/10.5935/2595o118.20190065. Disponível em: http://www. scielo.br/scielo.php?script=sci_arttext\&pi$\mathrm{d}=$ S2595-31922019000400356\&lng=en\& nrm=iso. Acesso em: 16 jul. 2020.

BARBOSA, R. E. C.; ASSUNÇÃO, A. da A.; ARAÚJO, T. M. de. Distúrbios musculoesqueléticos em trabalhadores do setor saúde de Belo Horizonte, Minas Gerais, Brasil. Cadernos de Saúde Pública, Rio de Janeiro, v. 28, n. 8, p. 1569-158o, ago. 2012. DOI: https:// doi.org/10.159o/So102-311X2012000800015 Disponível em: http://www.scielo.br/scielo.php?script $=$ sci_arttext $\&$ pid $=$ So102 -311 $\mathrm{X} 2012000800015 \& \operatorname{lng}=\mathrm{en} \& \mathrm{nrm}=\mathrm{iso}$. Acesso em: 16 jul. 2020.

BRASIL. Ministério da Saúde. Secretaria de Atenção à Saúde. Política Nacional de Humanização PNH. Brasília: Ministério da Saúde, 2013.

BRASIL. Ministério da Saúde. Secretaria de Atenção à Saúde. Departamento de Atenção Básica. Política nacional de práticas integrativas e complementares no SUS: atitude de ampliação de acesso. Brasília: Ministério da Saúde, 2015.

BRASIL. Ministério da Saúde. Fiocruz. Saúde mental e atenção psicossocial na pandemia
Covid-19: recomendações aos psicólogos para atendimentos online. Brasília: Ministério da Saúde 2020a. Disponível em: http:// renastonline.ensp.fiocruz.br/sites/default/ files/arquivos/recursos/saude-e-mental-e-atencao-psicossocial-na-pandemia-covid19-recomendacoes-aos-psicologos-para-o-atendimento-online-1.pdf. Acesso em: 16 jul. 2020.

BRASIL. Ministério da Saúde. Protocolo de manejo clínico do coronavírus (Covid-19) na Atenção Primária à Saúde. Brasília: Ministério da Saúde, 202ob. Disponível em: https://portalarquivos.saude.gov.br/images/ pdf/2020/April/o8/20200408ProtocoloManejo-vero7.pdf. Acesso em: 16 jul. 2020.

CRUZ, R. M. et al. COVID-19: emergência e impactos na saúde e no trabalho. Revista Psicologia: Organizações e Trabalho, Brasília, v. 20, n. 2, p. I-III, jun. 2020. Disponível em: http://pepsic.bvsalud.org/scielo.php?script=sci_arttext\&pid $=$ S1984-66572020000200001. Acesso em: 13 jul. 2020.

DO VALE, E. et al. Reorganização da Rede de Atenção à Saúde para o enfrentamento da Covid-19 no município de Canaã dos Carajás, Pará. APS em Revista, Belo Horizonte, v. 2, n. 2, p. 83-90, 9 jun. 2020. DOI: https://doi.org/10.14295/aps.v2i2.101.

HELIOTERIO, M. C. et al. COVID-19: por que a proteção da saúde dos trabalhadores e trabalhadoras da saúde é prioritária no combate à pandemia? SciELO 
Preprints. DOI: https://doi.org/10.159o/ SciELOPreprints.664. Disponível em: https://preprints.scielo.org/index.php/scielo/preprint/view/664/855. Acesso em: 16 jul. 2020.

HOLLIDAY, Oscar J. Para sistematizar experiências. 2. ed. Brasília: MMA, 2006.

JACKSON FILHO, J. M. et al. A saúde do trabalhador e o enfrentamento da Covid-19. Revista Brasileira de Saúde Ocupacional, São Paulo, v. 45, e14, 2020. DOI: https://doi. org/10.159o/2317-6369edoooo120. Disponível em: https://www.scielo.br/pdf/rbso/ v45/2317-6369-rbso-45-e14.pdf. Acesso em: 13 de julho de 2020.

JALES, R. D. et al. Auriculoterapia no cuidado da ansiedade e depressão. Revista de Enfermagem UFPE (on-line), [S.l.], v. 13, jun. 2019. ISSN 1981-8963. DOI: https://doi. org/10.5205/1981-8963.2019.240783. Disponível em: https://periodicos.ufpe.br/revistas/revistaenfermagem/article/view/240783. Acesso em: 30 jul. 2020.

OPAS. ORGANIZAÇÃO PAN-AMERICANA DE SAÚDE. Departamento de Evidência e Inteligência para Ação em Saúde. Entenda a infodemia e a desinformação na luta contra a Covid19. Página informativa n. 5, 2020. Disponível em: https://iris.paho. org/bitstream/handle/10665.2/52054/Factsheet-Infodemic_por.pdf?sequence $=14$.

Acesso em: 17 jun. 2020.

PRADO, A. D.; PEIXOTO, B. C.; DA SILVA, A. M. B.; SCALIA, L. A. M. A saúde mental dos profissionais de saúde frente à pandemia do Covid-19: uma revisão integrativa. Revista Eletrônica Acervo Saúde, n. 46, p. e4128, 26 jun. 2020. DOI: https://doi. org/10.25248/reas.e4128.2020.

RECIFE. Secretaria de Saúde do Recife. Protocolo de Assistência e Manejo Clínico da Covid-19 na Atenção Primária à Saúde do município de Recife. Versão 1. Recife, PE, mar. 202Oa.

RECIFE. Secretaria de Saúde do Recife. Atualização do Protocolo de Assistência e Manejo Clínico da Covid-19 na Atenção Primária à Saúde do município de Recife. Versão 4. Recife, PE, maio, 202ob.

RIBEIRO, M. et al. (RE)Organização da Atenção Primária à Saúde para o enfrentamento da Covid-19: Experiência de Sobral-CE. APS em Revista, Belo Horizonte, v. 2, n. 2, p. 177-188, 8 jun. 2020. DOI: https:// doi.org/10.25248/reas.e4128.2020.

SANTOS, A. Escuta qualificada como ferramenta de humanização do cuidado em saúde mental na Atenção Básica. APS em Revista, Belo Horizonte, v. 1, n. 2, p. 170-179, 24 jul. 2019. DOI: https://doi.org/10.14295/ aps.vii2.23.

SCHIMIDT, B. et al. Impactos na saúde mental e intervenções psicológicas diante da pandemia do novo coronavírus (Covid-19). SciELO Preprints. Disponível em: https:// preprints.scielo.org/index.php/scielo/preprint/view/58/69. Acesso em: 16 jul. 2020.

SBMFC. SOCIEDADE BRASILEIRA DE MEDICINA DE FAMÍLIA E COMUNIDADE. Recomendações da SBMFC para a APS durante a pandemia de Covid-19. Disponível em: https://redeaps.org.br/ wp-content/uploads/2020/05/Recomendac\%CC\%A70\%CC\%83es-da-SBMFC-para-a-APS-durante-a-Pandemia-de-COVID-19-1.pdf. Acesso em: 16 jul. 2020.

SOEIRO, R. E. et al. Atenção Primária à Saúde e a pandemia de Covid-19: reflexão para a prática. InterAmerican Journal of Medicine and Health, São Leopoldo, v. 3, p. e202003010, 2020. DOI: https:// doi.org/10.31005/iajmh.v3io.83. Disponível em: https://www.iajmh.com/iajmh/article/view/83/109. Acesso em: 16 jul. 2020.

Texto submetido em: 31 jul. 2020. Aprovado em: 8 out. 2020. QUIRINO, Túlio Romério Lopes; ROCHA, Luana Padilha da; CRUZ, Maria Soraida Silva; MIRANDA, Bruna Leitão; ARAÚJO, Janaína Gabriela Coêlho de; LOPES, Robélia do Nascimento; GONÇALVES, Suênia Xavier. Estratégias de cuidado à saúde mental do trabalhador durante a pandemia da Covid-19. Estudos Universitários: revista de cultura, Recife, v. 37, n. 1/2, p. 172-191, dez. 2020. ISSN Edição Digital: 2675-7354. 